\title{
Whole exome sequencing in inborn errors of immunity: use the power but mind the limits
}

\author{
Giorgia Bucciol/ ${ }^{\mathrm{a}}$, Erika Van Nieuwenhove ${ }^{\mathrm{b}, \mathrm{c}, \mathrm{d}}$, Leen Moens ${ }^{\mathrm{a}}$, \\ Yuval Itan ${ }^{\mathrm{e}, \mathrm{f}, *}$, and Isabelle Meyts ${ }^{\mathrm{a}, \mathrm{d}, *}$
}

\begin{abstract}
Purpose of review
Next-generation sequencing, especially whole exome sequencing (WES), has revolutionized the molecular diagnosis of inborn errors of immunity. This review summarizes the generation and analysis of nextgeneration sequencing data.
\end{abstract}

\section{Recent findings}

The focus is on prioritizing strategies for unveiling the potential disease-causing variant. We also highlighted oversights and imperfections of WES and targeted panel sequencing, as well as the need for functional validation.

\section{Summary}

The information is crucial for a judicious use of WES by researchers, but even more so by the clinical immunologist.

\section{Keywords}

inborn errors of immunity, next-generation sequencing, primary immunodeficiency, whole exome sequencing

\section{INTRODUCTION}

Inborn errors of immunity are a heterogeneous group of genetically determined diseases caused by defects in one or more components of immunity. These defects can manifest as increased susceptibility to infection, autoimmunity, autoinflammation, allergy, and malignancy or a combination of these [1]. In the last decade, advances in human genetics and bioinformatics have permitted an acceleration in the discovery of new genes associated with known or new monogenic diseases, with the greatest leap determined by the implementation of next-generation sequencing (NGS; reviewed in [2-4]). This is particularly relevant for inborn errors of immunity, where since 2010 a hundred new genes have been reported, of the over 300 genes so far described $\left[1,5^{*}, 6\right]$. Inborn errors of immunity are likely to be monogenic and display genetic pleiotropy (different clinical and immunological phenotypes originate from mutations of the same gene) and genetic heterogeneity (mutations in different genes give rise to the same phenotype) $[6,7 "-]$. For these reasons, NGS has accelerated the unraveling of these disorders at the genetic level. Incomplete penetrance is also a common feature of inborn errors of immunity. This can originate from oligogenic/polygenic models of disease, which are sometimes difficult to characterize. Epigenetic or environmental factors (e.g., previous encounter with a pathogen) may also play a role [8]. In this complex context, only in selected cases does Sanger sequencing of single genes lead to a definitive diagnosis. Moreover, many novel genetic etiologies are yet to be unraveled and many new phenotypes are likely to be ascribed to known genotypes [9].

The aim of this review is to provide information about the technical aspects of NGS and to illustrate its advantages and drawbacks in the setting of inborn errors of immunity. In particular, we will focus on some shortcomings of whole exome sequencing (WES).

${ }^{a}$ Childhood Immunology, Department of Microbiology and Immunology, KU Leuven, Belgium, 'baboratory of Genetics of Autoimmunity, Department of Microbiology and Immunology, KU Leuven, Belgium, ${ }^{\mathrm{C} V I B}$ Center for Brain and Disease Research, Leuven, Belgium, 'Department of Pediatrics, University Hospitals Leuven, Belgium, ${ }^{\mathrm{e} T h e}$ Charles Bronfman Institute of Personalized Medicine and 'Department of Genetics and Genomic Sciences, Icahn School of Medicine at Mount Sinai, New York, USA

Correspondence to Isabelle Meyts, MD, PhD, Department of Microbiology and Immunology, KU Leuven, Herestraat 49, bus 7003, 3000 Leuven, Belgium. Tel: +32 016343841; fax: +32 016343842;

e-mail: isabelle.meyts@uzleuven.be

*Yuval Itan and Isabelle Meyts equally contributed to this article.

Curr Opin Allergy Clin Immunol 2017, 17:000-000

DOI:10.1097/ACl.0000000000000398 


\section{KEY POINTS}

- NGS is revolutionizing the diagnostics and the research of Mendelian diseases.

- Although NGS is called an unbiased diagnostic approach, its value stands or falls with the genetic hypothesis.

- Caution is needed during the filtering phase of NGS analysis, to prevent the inclusion of false positives and exclusion of false negatives.

- The incomplete coverage of the genome, the difficulty in analyzing CNVs, segmental duplications and pseudogenes by WES, and the weaknesses of the prediction methods used to filter NGS data, need to be considered.

- When a mutation in a novel gene or a novel mutation in a known gene is detected, a causative link with the clinical phenotype must be established by meticulous functional validation.

\section{NEXT-GENERATION SEQUENCING}

NGS is a high-throughput sequencing method in which thousands or millions of small DNA fragments are sequenced in parallel [2]. Starting from the patient's genomic DNA, a sequencing library is prepared by random fragmentation of the DNA into short strands that are then fused with adaptors. These fragments are amplified and sequenced in parallel multiple times, usually at least 20 times for any nucleotide, to attain sufficient read depth. The obtained sequences are then mapped to a reference human genome and the differences between the patient's DNA and the reference are 'called' as variants (Fig. 1). A read depth of at least 20 is essential to discriminate sequencing errors from true variants. These variants must be analyzed in the setting of the patient's clinical and cellular phenotype and pedigree to disregard irrelevant variants and to instead detect known or novel mutations in known disease-causing genes or in potentially novel candidate genes [5"].

NGS can be used to sequence the entire genome [whole genome sequencing (WGS)], a targeted panel such as the WES, or a more limited set of genes of interest. The exome is defined as all the exons for about 20000 protein-coding genes and for microRNA, small nucleolar RNA, and large intergenic noncoding RNA genes, constituting about $1 \%$ of

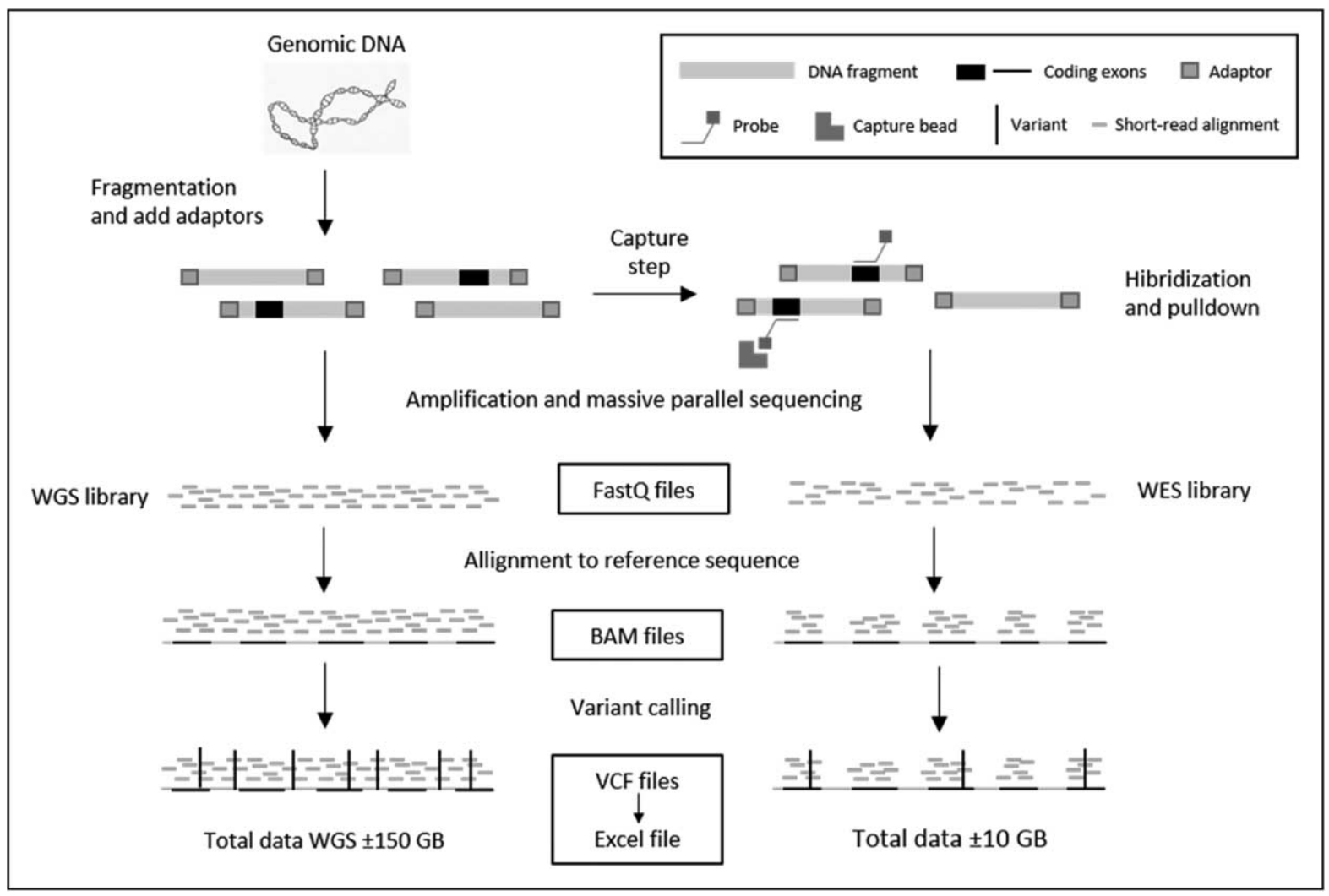

FIGURE 1. The scheme illustrates the process of WGS and WES. The files that are finally derived from the analysis are of three types: FASTQ files, including all the raw data; BAM files, for the alignment to the reference sequence; and VCF files, subsequently convertible to Excel files, for the variant calling. WES, whole exome sequencing; WGS, whole genome sequencing. Adapted with permission [5"]. 
the human genome [10]. In the construction of the DNA library, WES and panel sequencing require enrichment in exons through hybridization with target-specific probes and PCR amplification of the selected regions [11]. This additional step generates bias at different levels: as the enrichment process is not perfectly homogeneous and there is an $a$ priori reference bias with the choice of probes, some exons are underrepresented or missing in the library; moreover, the PCR amplification step can introduce errors in the sequences of nucleotides because of polymerase errors, guanine-cytosine related errors, stochastic errors and template switch errors $\left[5^{\prime}, 12\right]$. Accordingly, WGS was shown to be more powerful than WES for detecting exome variants (single nucleotide variants) as well as small insertions/deletions (indels) and copy number variations (CNVs). WGS provides a much more uniform distribution of sequencing-quality parameters and a more homogeneous coverage of the genome [13].

\section{WHOLE EXOME SEQUENCING AND WHOLE GENOME SEQUENCING ANALYSIS PIPELINE}

WES and WGS raw data output files generated by highthroughput sequencing instruments, such as the Illumina Genome Analyzer (San Diego, California, US), are in FASTQ format. Burrows-Wheeler Aligner [14] is often used for subsequent alignment of the reads to the reference genome, resulting in Binary Alignment Map files. In the next step a Variant Call Format file, containing the chromosome position and nucleotide change for each variant, is created using for instance Genome Analysis Toolkit [15]. Finally, the Variant Call Format files (already containing information on the sequencing quality and read depth) can be further annotated with different tools available online, with the purpose of simplifying data analysis. Annovar [16], SnpEff [17], Variant Effect Predictor [18], and others add information about a specific variant based on available data on its position in the gene, the amino acid change, the molecular effect of the variant (missense, nonsense, and so on), the allele frequency in different populations, and the protein damage prediction. Encyclopedia of DNA Elements (ENCODE) is a useful resource for annotating intergenic variants [19]. The allele frequency of the variant is a fundamental tool to filter out common polymorphisms and can be derived from publicly available databases $\left[20^{-*}, 21\right]$. BioGPS [22] and Protein Atlas [23] show the expression of the gene and the protein for which it encodes in human tissues and cell lines, whereas Evolutionary Conserved Regions Browser [24] and phylogenetic $p$ (Phylop) score [25] display the conservation of genes and DNA sequences across species.
Relevant information about genes and their encoded products are collected in integrative databases, such as GeneCards (www.genecards.org) [26]. Finally, additional information can be gained by combining different annotations that predict deleteriousness/ protein impact, such as in the combined annotation-dependent depletion (CADD) score [27]. Relevant annotations can be chosen and implemented into a personalized pipeline, based on the research or diagnostic laboratory's specific needs. Some of the most commonly used annotations are further discussed in the chapter 'Finding the disease-causing variant.'

\section{CHOOSING THE APPROPRIATE NEXT- GENERATION SEQUENCING STRATEGY FOR INBORN ERRORS OF IMMUNITY}

Compared with WGS, WES and targeted panel sequencing have the advantage of being cheaper and of generating a much smaller set of data to analyze [11,28-30]; the drawbacks include an incomplete coverage of the genome (WES misses most intronic mutations and some mutations in nonprotein-coding genes), and technical problems related to the capture step. For targeted panels, the ulterior restriction of the analysis to genes already described in connection with disease is the major limitation. As the rate of description of novel genes causing inborn errors of immunity is now, on average 10/year, gene panels become in fact quickly obsolete [5"]. The Mendeliome, a panel covering all known genes causing Mendelian disorders, has been proposed in 2015 as a cheaper and more manageable diagnostic tool compared to WES. However, the same reservations as for targeted panel analysis hold true [31]. For these reasons, from a technical point of view WGS is at present the most reliable approach for the discovery of known and novel mutations in known and novel genes, at the expense of sequencing cost and time needed to analyze the much wider dataset. However, as $85 \%$ of mutations generating Mendelian disorders are estimated to be located in coding regions of the genome, WES remains a highly valuable technique both for clinical diagnostics and for the research of novel diseaseassociated genes [32]. Clinical exome sequencing is now being offered by diagnostic labs over the world. In this setting, a disease-specific panel-like analysis of the exome data is performed and allows for a more efficient approach in genetic diagnosis.

\section{FINDING THE DISEASE-CAUSING VARIANT}

WES generates on average 20000 high-quality coding variants. To minimize the presence of common variants and maximize the inclusion of probable 


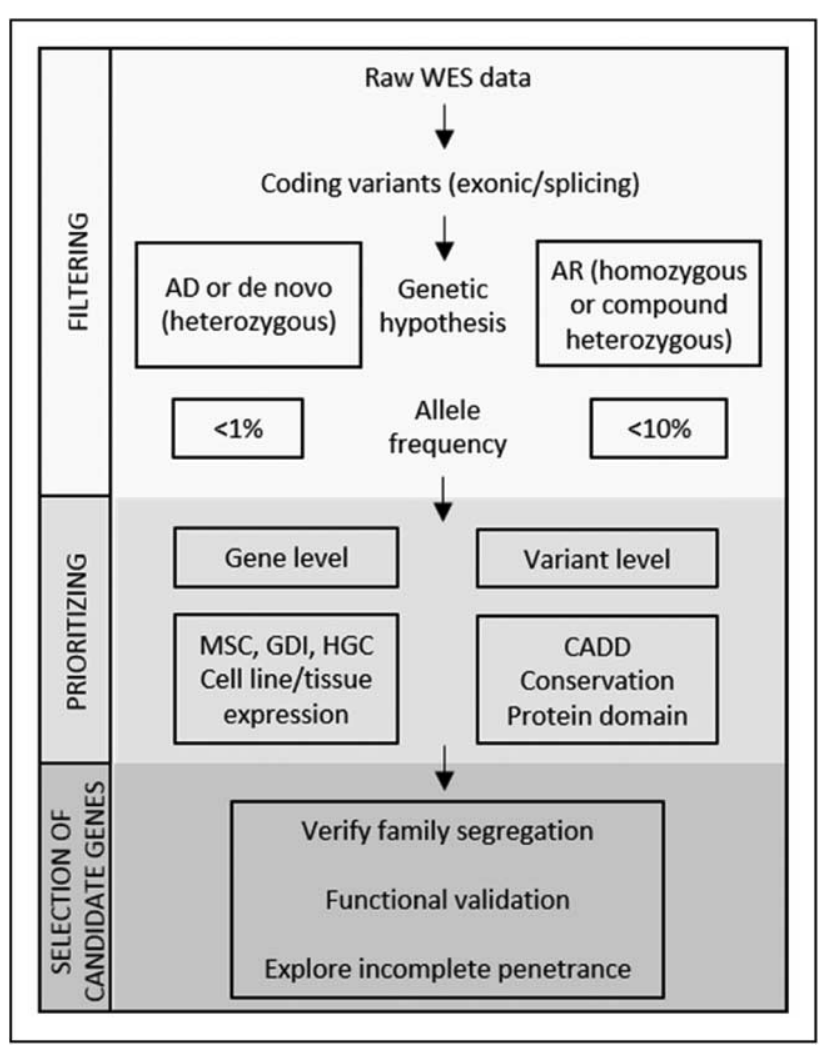

FIGURE 2. Flow chart depicting the general filtering method to screen whole exome data for disease-causing mutations. $A D$, autosomal dominant; $A R$, autosomal recessive; $C A D D$, combined annotation-dependent depletion; GDI, gene damage index; HGC, human gene connectome; MSC, mutation significance cutoff; WES, whole exome sequencing.

disease-causing mutations, a gene-level and a variant-level filtering approach are applied (Fig. 2) [3].

The first crucial step is the generation of a genetic hypothesis that takes into consideration the potential mode of inheritance, the severity of the phenotype, the penetrance, and so on. The presence of multiple affected siblings in a family with healthy ancestors is suggestive of autosomal recessive inheritance, so homozygous (especially in the case of consanguineous unions) or compound heterozygous variants should be prioritized. The inclusion of multiple affected and unaffected siblings is extremely useful to discern the potential disease-causing variant. Nevertheless, the possibility of autosomal dominant, X-linked recessive inheritance, or de novo mutations should not be neglected in consanguineous unions. Trio analysis, in which the index patient and parents are sequenced simultaneously, is particularly efficient to pinpoint disease-causing de novo mutations; it is also very informative in the detection of homozygous and compound heterozygous mutations. Severe phenotypes in single patients should be investigated in particular for de novo, homozygous, or compound heterozygous variants, although in the case of incomplete penetrance this approach could exclude the causal mutation. Finally, autosomal dominant inheritance implies that a heterozygous mutation is disease causing. In accordance with the suspected inheritance, a hypothesis must be formed on the detected variants. Heterozygous dominant variants are generally considered gain-of-function (GOF) mutations, where the encoded protein acquires a new function or expression pattern. However, dominant loss-of-function (LOF) variants can also occur through haploinsufficiency, where a reduction of gene function by $50 \%$ is sufficient to result in disease, or by a dominant negative effect, where the mutant gene product acts antagonistically to the wild-type protein function [ $\left.7^{-"}\right]$. This can occur, for instance, if the mutant protein hampers multimer formation. Disease-causing compound heterozygous or homozygous variants are presumed LOF mutations.

The following criteria represent a general guideline to consider a gene to harbor potential diseasecausing variants, even if they are not always valid and several counter examples can be found: it encodes a protein involved in a pathway related to the phenotype; it is expressed in the cells and tissues of interest; it is intolerant to mutations in the general population, indicating that mutations in that gene have a higher probability of being deleterious. There are databases reporting the expression of human genes across human cell lines, cell types, tissues, and organs $[22-23,26]$. It is reasonable to hypothesize that the gene of interest is expressed in the affected cell or tissue, although mutations in ubiquitously expressed genes can result in a phenotype that is highly tissue specific [33]. The connection of the candidate gene with a certain pathway or protein that is known to be related with the disease can be tested using the Human Gene Connectome [34-35], which is based on protein-protein interactions and depicts the biological distance and route between two genes [the gene(s) connecting them]. The gene damage index (GDI) is a tool made publicly available at http://lab.rockefeller.edu/casanova/GDI in 2015 to filter candidates at the gene level, complementing available variant-level metrics [36]. It is defined as the cumulative mutational damage to a particular human gene in the general population based on data from the 1000 Genomes Project [21], and it can be used to remove genes that are highly mutated in the general population and, therefore, less likely to cause severe disease [36]. Other methods, such as residual variation intolerance score, are complementary by assessing the 
likelihood of genes to be pathogenic based on their intolerance to mutations [37].

At the variant level, the allele frequency, based on different public databases in accordance with the ethnicity of the studied kindred, is the main filter to substantially reduce the number of variants. The hypothesized inheritance model dictates the frequency cutoff that should be applied. In general, heterozygous variants with a frequency in the general population of more than $1 \%$ are considered polymorphisms, with at most a weak modulating effect on disease. In the context of monogenic disorders, for autosomal dominant transmission a frequency cutoff of less than 1\% is thus applied. For autosomal recessive disorders, instead, a higher frequency cutoff is recommended to minimize false negatives: to obtain a frequency cutoff of $1 \%$ for the homozygous state, we need to combine two parental alleles with a frequency of $10 \%$, respectively, to respect Hardy-Weinberg equilibrium. Exceptions can derive from population genetics phenomena, such as founder effect, resulting in a high carrier frequency (such as in Adenosine Deaminase 2 (ADA2) deficiency (38)], or evolutionary adaptation [e.g., Familiar Mediterranean Fever gene (MEFV) mutations that provide a selective advantage against Yersinia pestis infection (39)]. These cutoffs can be further reduced based on knowledge on the disease prevalence and on the predicted clinical penetrance. In the research setting, more strict cutoffs are used to screen for novel disease-causing variants, which usually have a very low allele frequency. Coding variants that are synonymous, that is, variants that do not cause a change in the amino acid at that position, and noncoding variants that do not affect splicing, are usually excluded. This step significantly reduces the data load to be analyzed, as in most cases synonymous variants are silent or do not cause a change to the protein. Rarely, synonymous variants alter disease susceptibility or cause disease, for example, by impairing splicing, or by affecting mRNA stability or protein folding (reviewed in [40]).

Various algorithms that attempt to predict the functional effect and assess the conservation/intolerance of a missense variant are publicly available, such as the Phylop score [25], the Genomic Evolutionary Rate Profiling programs [41], Sorting Intolerant from Tolerant (SIFT) [42], or Polymorphism Phenotyping v2 (PolyPhen-2) [43]. They are combined with information from other annotations to create the CADD score [27]. CADD is more precise in predicting the deleteriousness of both proteinaltering and regulatory variants. CADD scores range from 0 to 99 with increasing deleteriousness, and 15 has been proposed as a standard cutoff for all human genes. However, like other prediction tools, CADD is not flawless [27]. For example, reliable data input is lacking for intronic variants and it has been shown that CADD score has a high false negative rate when using a fixed cutoff [44"]. A more precise assessment of the variant's effect at the gene level can be obtained by using the mutation significance cutoff (MSC), a quantitative method that provides genespecific phenotypic impact cutoff values, and represents the lowest expected clinically/biologically relevant CADD (as well as PolyPhen-2 and SIFT) cutoff value for a specific gene [44"]. The combination of both variant and gene-level approaches, for example, by considering the GDI, the MSC and the CADD score together, allows better prioritization, with less devaluation of false negative variants $\left[5^{*}, 44^{\prime \prime}\right]$. In other words: a benign variant (with a CADD below the MSC of that gene) with a high GDI is expected to have the lowest phenotypic impact. A variant with a CADD score above the gene-specific MSC in a gene displaying low or medium GDI and which is also biologically close to known diseasecausing genes, can be expected to have the greatest phenotypic impact.

\section{VALIDATION OF THE FUNCTIONAL IMPACT OF A VARIANT}

No prediction tool has the power to prove the pathogenicity of a variant. This is why, upon discovery of a potential new disease-causing gene or variant, it is fundamental to experimentally ascertain the causal connection between the variant and the clinical and immunological phenotype, to prevent ascribing to a false-positive result a diseasecausing effect [7"']. A multidisciplinary team meeting involving the clinician (scientist), genetic counsellor, bioinformatician, and clinical and nonclinical researcher is optimal for efficient prioritizing of variants that need further validation. Especially for single-patient genetic studies, solid evidence to show that the genetic variant is responsible for the destruction, impairment of function or alteration of expression or function of the protein product is necessary [4]. First, the effect of the variant on RNA or protein expression (for instance, by Western Blot or flow cytometry) must be investigated. In the case of unaltered protein expression, altered function can lead to disease. Functional studies are therefore a crucial step. For instance, in the study of mutations in genes affecting the type I interferon pathway, the in-vitro assay of viral load in cell lines relevant to the phenotype can be pursued. In case of a de novo mutation, different cell types should be tested to exclude a somatic mutation. Finally, for LOF mutations the cellular phenotype should be rescued by transduction with the 
wild-type allele, whereas for GOF mutations rescue should be obtained by knockdown/knockout or correction of the mutant allele [4]. The functional confirmation of causality represents the most difficult and time-consuming part in the characterization of new disease-associated genes or mutations, but remains essential for the scientific validation of the findings and hence also for the diagnostic value of a reported variant. Reports of novel 'mutations', even if in genes known to cause inborn errors of immunity, should be interpreted with caution if the validation step is missing.

\section{PITFALLS OF NEXT-GENERATION SEQUENCING}

NGS, and WES in particular, present some common drawbacks and technical difficulties that we are now going to outline.

\section{Incomplete coverage of the genome by whole exome sequencing}

The major pitfall of WES is that, by design, its coverage excludes noncoding exons, for example, RNA genes, and intronic/intergenic regions, although they can harbor disease-causing mutations. Examples are Roifman syndrome, caused by compound heterozygous mutations in the RNA U4 small nuclear AT-AC form (RNU4ATAC) gene, encoding the minor spliceosomal small nuclear RNA U4atac, and X-linked reticulate pigmentary disorder (X-linked PDR), caused by a hemizygous (in men) or heterozygous (in women, who have a milder phenotype with only pigmentary anomalies) intronic mutation in the DNA polymerase alpha gene (POLA1), affecting splicing. Both diseases were genetically characterized thanks to WGS, performed on the clinically affected patients [45-46]. In addition to noncoding exons and introns, a large number of exons (up to $6 \%$ of the total, in Belkadi et al. [13]) from protein-coding genes are yet not covered in exome kits, even those included in the Consensus Coding DNA Sequence database. Moreover, it has been shown how, even in targeted regions, many high-quality true positive variants are missed by WES compared with WGS, whereas the variants missed by WGS but detected by WES are mostly false positives [13].

\section{When quantity matters}

CNVs are large segments of DNA that by duplication or deletion are present in a variable number in the genome of different individuals (Fig. 3). They can be disease-associated or simple polymorphisms, and

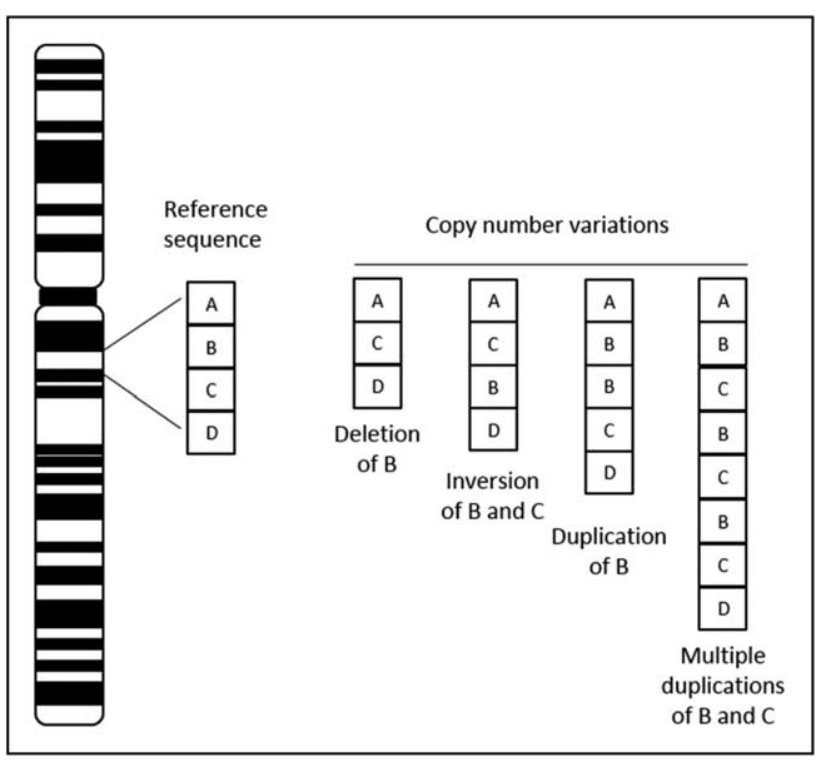

FIGURE 3. The figure illustrates the types of copy number variations that can arise from deletions, inversions, and duplication of segments of DNA. The squares marked by $A, B, C$, and D represent large regions of genomic DNA.

are increasingly recognized to play a role in interindividual variability [47-49]. Segmental duplications, or low copy repeats, are either inter or intrachromosome duplications of highly identical stretches of DNA, from 1 to $400 \mathrm{~kb}$ in length [4951]. Segmental duplications have played a major role in human evolution from primates and are much more represented in our genome compared with chimpanzee, with 138 gene duplications specific to humans identified as human-chimpanzee divergence [52-53]. In fact, approximately 5\% of the human genome was shown to consist of segmental duplications of $10 \mathrm{~kb}$ or larger [54], and up to $97 \%$ of genes with a CNV correspond to known segmental duplications, thus illustrating a propensity for chromosomal rearrangements in these regions [48]. A change in copy number of a gene or a disruption of its integrity can arise from nonallelic homologous recombination of repeated sequences (Fig. 4). It is the cause of many genomic disorders, including X-linked ichthyosis, $\alpha$-Thalassemia, and DiGeorge syndrome [49-51,54].

Large structural variations of the DNA are traditionally analyzed by karyotyping and by array-based comparative genomic hybridization (array CGH). Standard karyotyping is based on microscopical observation of the chromosome and can, therefore, only detect numerical or structural aberration of at least 5-10 million base pairs (Mb). In-situ fluorescence hybridization employs labeled DNA probes to detect smaller chromosomal rearrangements at a resolution of $1-5 \mathrm{Mb}$, but it can be used only when 


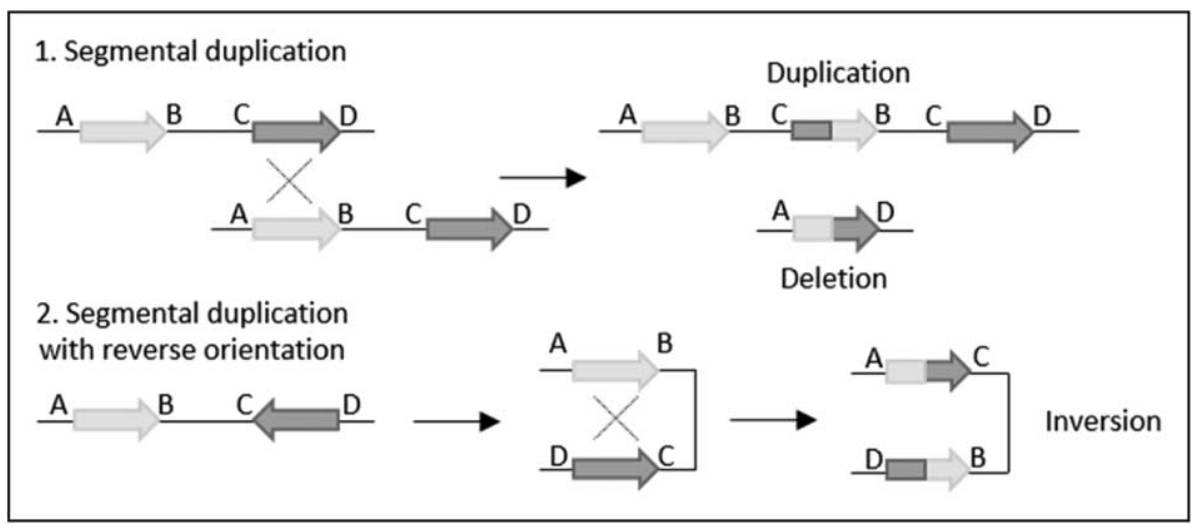

FIGURE 4. Different types of nonallelic homologous recombination between segmental duplications (red and blue arrows represent the same duplicated region). (1) Recombination between homologous sequences on different DNA strands can generate either a duplication or a deletion of the region between the segmental duplications. (2) Recombination between homologous sequences with reverse orientation leads to inversion of the recombined fragments.

the specific aberration is already known [55]. Array CGH instead allows us the identification of smaller variations (from $\sim 200 \mathrm{bp}$ ) by hybridizing the sample DNA together with a reference DNA to the normal human genome, and then measuring the differences between sample and reference [56]. An example of the application of array CGH to the diagnostic of inborn errors of immunity is the description by Kazenwadel et al. [57] of a large genomic deletion encompassing GATA binding protein 2 (GATA2) gene in a family with myelodysplastic syndrome.

When approached by WES, segmental duplications and large structural variations such as CNVs present technical difficulties at two levels.

\section{Copy number variations}

CNVs can significantly contribute to the cause of Mendelian disorders. For this reason, it is important to find strategies that allow them to be detected by WES, even if calling CNVs from short read sequence data can be very challenging. High-resolution mapping of copy number alterations is already very effective for WGS, whereas WES is currently not very reliable, as a result of the fragmentation of the captured exons and the extension of most CNVs beyond the covered amplicons [13,48]. Different strategies are being tested to improve CNVs detection by WES; among them, read depth analysis has proven particularly effective [58",59]. This approach assumes a random distribution in mapping depth. It compares the number of reads mapping to a certain chromosome region with the prediction by a statistical model, calling deviations from the model as CNVs $\left[58^{*}, 60\right]$. This method yields better results when the ratio of read counts between a test sample and a control sample is used instead of a single sample analysis, to compensate for the technical variability in capture efficiency and other sequencing biases $\left[58^{*}, 60\right]$. The different algorithms and platforms available for CNVs analysis are reviewed and compared in Kadalayil et al. [58"].

\section{Segmental duplications}

In the case of segmental duplications, the generation of relatively short sequence reads required by WES makes it impossible to distinguish between a duplicated gene and its parent gene during their alignment to the reference sequence. This prompted many researchers to systematically exclude these duplication-rich genomic regions from their downstream analysis. Caution is instead needed, as the largest segmental duplications exhibit an interindividual variability of only $3 \%$ and duplications do not preclude the occurrence of highly damaging mutations [48]. We draw on two examples from the field of inborn errors of immunity. Sequencing of the gene encoding filaggrin ( $F L G$ ) proved challenging because of the large exon 3 which includes 10-12 highly identical full tandem repeats [61]. However, even nonsense and frameshift mutations in these imperfect C-terminal repeats are pathogenic for ichthyosis vulgaris and predispose to eczema and allergic diseases [61]. In our quest for a causative gene defect in a patient with severe atopic dermatitis, recurrent skin infections and other features reminiscent of hyper-IgE syndrome including a serum IgE of $30000 \mathrm{kU} / \mathrm{l}$, a pathogenic homozygous mutation in FLG was filtered out because it was located in a segmental duplication area. Autosomal dominant combined immunodeficiency with lymphoproliferation because of mutations in the Phosphatidylinositol 3-kinase catalytic delta (PIK3CD) gene, in particular the known pathogenic E1021K substitution, may also be missed because of a duplicated 
region in exon 22 [21], as was our experience in a boy for whom we did trio exome sequencing but only found the causative gene via single gene Sanger sequencing after carefully reconsidering the clinical phenotype. Hence, it is not safe to automatically exclude segmental duplications when analyzing WES data. This case also shows the crucial role of the astute clinician in guiding and navigating genetic analysis.

\section{Pseudogenes}

Pseudogenes are segments of genomic DNA that are related to normal genes but not functional. They are characterized by sequence homology with a parent gene and the loss of protein-coding ability. This is the result of two main mechanisms: the duplication of a gene followed by accumulation of mutations that cause the deactivation of the redundant copy, or the retrotransposition of mRNA back into the genome, generating a gene that is by default nonfunctional, lacking promoter regions (Fig. 5) [62]. The Inhibitor of kappa light polypeptide gene enhancer in B cells, kinase gamma (IKBKG)/NF-kB essential modulator (NEMO) gene, coding for NEMO, represents a significant example of pseudogene and segmental duplication. It is in fact part of a segmental duplication containing the functional gene and, in the opposite orientation, its partial pseudogene copy IKBKGP [63-64]. Nonallelic homologous recombination between the two highly similar regions can generate rearrangements both of the gene and the pseudogene, most often CNVs. When they involve the functional gene, they can be the cause of incontinentia pigmenti in affected women [63-64]. This peculiar genomic structure renders analysis of $I K B K G / N E M O$ by WES extremely difficult. In the alignment to the reference sequence, it is impossible to attribute with certainty reads from homologous regions to the gene or the pseudogene. This has important diagnostic consequences, as the same single nucleotide variant or indel is pathogenic when affecting the gene but benign in the pseudogene. A reliable method to assess whether a variant belongs to one or the other is long-range PCR, that allows discrimination between the two regions, thanks to specific primers [65].

\section{Gene and variant-level prediction methods}

At the gene level, the GDI and residual variation intolerance score methods are founded on the fair assumption that frequently mutated genes in the healthy population are less likely to be pathogenic for rare, inherited disorders such as inborn errors of immunity. They are efficient in eliminating these overrepresented highly mutated genes, containing missense, nonsense, or indel variants not considered detrimental biochemically or evolutionarily. In other words, they provide us with a tool to remove plausible false positive variants from NGS data. Focusing on GDI, the cutoff values proposed for the filtering process in the context of inborn errors of immunity are 12.41 for all disease-causing genes, and more specifically 13.36 and 9.49 , respectively, for autosomal recessive or autosomal dominant inheritance [36]. Despite the many advantages, it is prudent to consider its limitations. In the first place, to generate these cutoff values a false negative rate of 5\% was tolerated [36]. Therefore, variants in some disease-causing genes [including Dedicator of cytokinesis 8 (DOCK8), Interferon-induced helicase $\mathrm{C}$ domain-containing protein 1 (IFIH1), Lipopolysaccharide-responsive beige-like anchor protein (LRBA), and Ataxia-teleangectasia mutated $(A T M)]$ will be excluded from the downstream analysis. Furthermore, the GDI may be

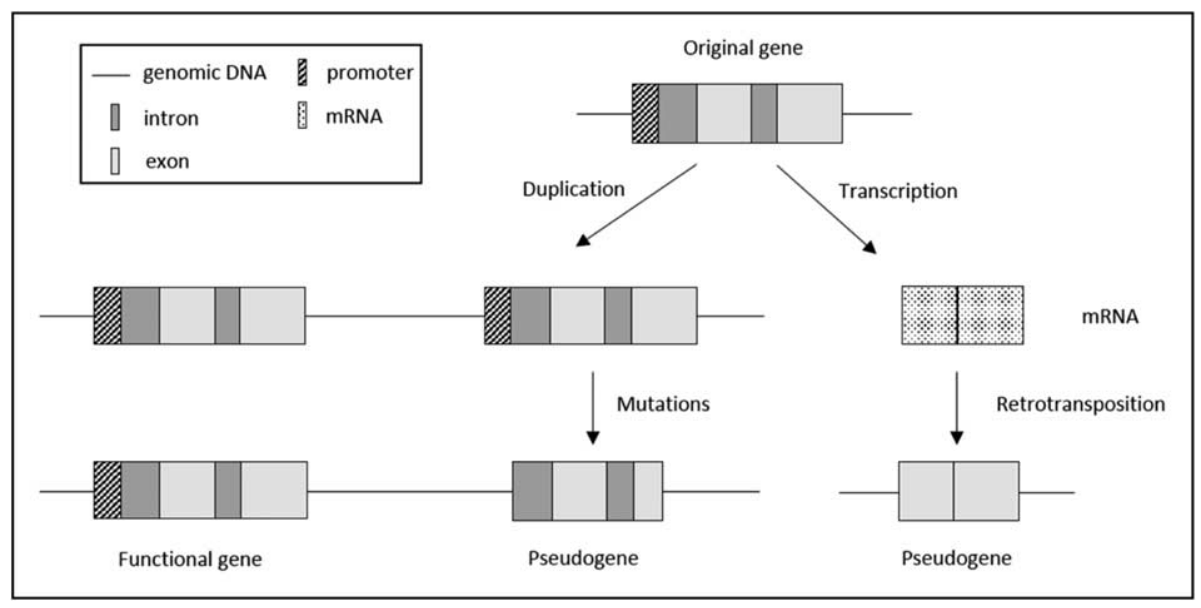

FIGURE 5. The figure illustrates two possible mechanisms of generation of pseudogenes: duplication of a gene followed by accumulation of deleterious mutations in the redundant copy, or retrotransposition of mRNA in the DNA, producing a nonfunctional pseudogene. 
population specific, and when performing genetic analysis it remains imperative to take these factors into account. Finally, the GDI does not compensate for $\mathrm{CNV}$ s present in these genes, which translates into a higher than expected GDI value. The gene $F L G$ is an example with a GDI value of 27.35 but a demonstrated role in severe eczema. This issue of adjusting the cutoffs to include as many true positives as possible, whereas trying to eliminate false negatives applies also to the variant-level prediction methods, such as allele frequency and CADD score. We recommend to either establish a false negative rate suitable for your personal objective or to merely use these tools in the prioritization of variants (what they were ultimately designed for), but not as a final cutoff.

\section{CONCLUSION AND FUTURE DIRECTIONS}

NGS has revolutionized the diagnostic and research approach to monogenic diseases, inborn errors of immunity in particular. For this reason, physicians treating the patients affected by these disorders wish to be familiar with these techniques, and know their strengths and weaknesses.

We have, here, reviewed the process of WGS and targeted panel sequencing, including WES; the steps required to find the disease-causing variant, starting from the genetic hypothesis steps and then proceeding with a gene-level and a variant-level approach; and finally some common pitfalls of these methods. Based on the growing expertise and growing technical opportunities now available in this field, both the exome kits as well as the annotations tools are becoming more and more precise in identifying inborn errors of immunity causative mutations, but we should be ready to revisit all assumptions in the screening pipeline when they fail to detect a pathogenic variant.

Thanks to continuous technologic advancements, the cost of WGS is rapidly decreasing and it will soon become as cheap as WES, with the advantage of being more accurate in detecting mutations. As a diagnostic approach, we therefore propose to first sequence a targeted panel of genes or the exome, then extending the investigation to the entire genome if no adequate candidate gene or variant is found. The biggest challenge will remain the validation of new candidate mutations, which is absolutely required to prove the disease-causing nature of a genetic variant [4].

Our ever-expanding molecular understanding of inborn errors of immunity is paving the way for targeted therapy, such as the use of Janus Kinase $1 / 2$ inhibitors in signal transducer and activator of transcription 1 (STAT1) GOF mutations or inhibitors of the PI3K catalytic subunit p1108 in PIK3CD
GOF mutations [66"'], gene therapy, and soon geneediting technology. In this way, WES will not only have revolutionized genetic diagnosis, but also medicine as a whole.

\section{Acknowledgements}

None.

\section{Financial support and sponsorship}

I.M. is supported by a KOF grant of the KU Leuven, by the Research Foundation - Flanders (FWO, grant number GOC8517N), and by the Jeffrey Modell Foundation. G.B. is supported by the Research Foundation Flanders (FWO, grant number ZKD2020-01-W01). L.M. is supported by the CSL Behring Chair in Primary Immunodeficiencies. E.V.N. is supported by the Research Foundation Flanders (FWO, doctoral grant strategic basic research number $1 S 22716 N)$.

\section{Conflicts of interest}

There are no conflicts of interest.

\section{REFERENCES AND RECOMMENDED}

\section{READING}

Papers of particular interest, published within the annual period of review, have been highlighted as:

- of special interest

m. of outstanding interest

1. Picard $\mathrm{C}, \mathrm{Al}-\mathrm{Herz} \mathrm{W}$, Bousfiha $\mathrm{A}$, et al. Primary immunodeficiency diseases: an update on the classification from the international union of immunological societies expert committee for primary immunodeficiency 2015. J Clin Immunol 2015; 35:696-726.

2. Shendure J, Ji H. Next-generation DNA sequencing. Nat Biotechnol 2008; 26:1135-1145.

3. Bamshad MJ, Ng SB, Bigham AW, et al. Exome sequencing as a tool for Mendelian disease gene discovery. Nat Rev Genet 2011; 12: $745-755$.

4. Casanova JL, Conley ME, Seligman SJ, et al. Guidelines for genetic studies in single patients: lessons from primary immunodeficiencies. J Exp Med 2014; 211:2137-2149.

5. Meyts I, Bosch B, Bolze A, et al. Exome and genome sequencing for inborn - errors of immunity. J Allergy Clin Immunol 2016; 138:957-969.

The article reviews the application of NGS to the diagnostics of inborn errors of immunity, illustrating the necessary steps and reporting all the relevant genes discovered by NGS.

6. Conley ME, Casanova JL. Discovery of single-gene inborn errors of immunity by next generation sequencing. Curr Opin Immunol 2014; 30:17-23.

7. Zhang Y, Su HC, Lenardo MJ. Genomics is rapidly advancing precision - medicine for immunological disorders. Nat Immunol 2015; 16:1001-1004.

The article reviews the application of NGS in immunological disorders with emphasis on the genetic hypothesis.

8. Platt C, Geha RS, Chou J. Gene hunting in the genomic era: approaches to diagnostic dilemmas in patients with primary immunodeficiencies. J Allergy Clin Immunol 2014; 134:262-268.

9. Kienzler AK, Hargreaves CE, Patel SY. The role of genomics in common variable immunodeficiency disorders. Clin Exp Immunol 2017; 188: 326-332.

10. Aken BL, Ayling $S$, Barrell D, et al. The Ensembl gene annotation system. Database (Oxford) 2016; 2016:; pii: baw093.

11. Ng SB, Turner EH, Robertson PD, et al. Targeted capture and massively parallel sequencing of 12 human exomes. Nature 2009; 461:272-276.

12. Kebschull JM, Zador AM. Sources of PCR-induced distortions in highthroughput sequencing data sets. Nucleic Acids Res 2015; 43:e143.

13. Belkadi $A$, Bolze $A$, Itan $Y$, et al. Whole-genome sequencing is more powerful than whole-exome sequencing for detecting exome variants. Proc Natl Acad Sci U S A 2015; 112:5473-5478.

14. Li H, Durbin R. Fast and accurate short read alignment with Burrows-Wheeler transform. Bioinformatics 2009; 25:1754-1760. 
15. McKenna A, Hanna M, Banks $E$, et al. The Genome analysis toolkit: a MapReduce framework for analyzing next-generation DNA sequencing data. Genome Res 2010; 20:1297-1303.

16. Yang $\mathrm{H}$, Wang K. Genomic variant annotation and prioritization with ANNOVAR and WANNOVAR. Nat Protoc 2015; 10:1556-1566.

17. Cingolani $P$, Platts $A, W a n g L L$, et al. A program for annotating and predicting the effects of single nucleotide polymorphisms, SnpEff: SNPs in the genome of Drosophila melanogaster strain w1118; iso-2; iso-3. Fly (Austin) 2012; 6:80-92.

18. Yen JL, Garcia S, Montana A, et al. A variant by any name: quantifying annotation discordance across tools and clinical databases. Genome Med 2017; 9:7.

19. Project ENCODE Consortium. An integrated encyclopedia of DNA elements in the human genome. Nature 2012; 489:57-74.

20. Lek M, Karczewski KJ, Minikel EV; Exome Aggregation Consortium. Analysis

.1. of protein-coding genetic variation in 60,706 humans. Nature 2016; 536:285-291.

The database represents the reference for allele frequency.

21. 1000 Genomes Project Consortium. Auton A, Brooks LD, Durbin RM. A global reference for human genetic variation. Nature 2015; 526: $68-74$.

22. Wu $\mathrm{C}$, Jin $\mathrm{X}$, Tsueng $\mathrm{G}$, et al. BioGPS: building your own mash-up of gene annotations and expression profiles. Nucleic Acids Res 2016; 44: D313-D316.

23. Uhlén $M$, Fagerberg L, Hallström BM, et al. Proteomics. Tissue-based map of the human proteome. Science 2015; 347:1260419.

24. Ovcharenko I, Nobrega MA, Loots GG, Stubbs L. ECR browser: a tool for visualizing and accessing data from comparisons of multiple vertebrate genomes. Nucleic Acids Res 2004; 32:W280-W286.

25. Pollard KS, Hubisz MJ, Rosenbloom KR, Siepel A. Detection of nonneutral substitution rates on mammalian phylogenies. Genome Res 2010; 20: 110-121.

26. Stelzer G, Rosen N, Plaschkes I, et al. The GeneCards suite: from gene data mining to disease genome sequence analyses. Curr Protoc Bioinformatics 2016; 54:1.30.1-1.30.33.

27. Kircher $M$, Witten $D M$, Jain $P$, et al. A general framework for estimating the relative pathogenicity of human genetic variants. Nat Genet 2014; 46:310-315.

28. Moens LN, Falk-Sorqvist $E$, Asplund AC, et al. Diagnostics of primary immunodeficiency disease: a sequencing capture approach. PLoS One 2014; 9:e114901.

29. Nijman IJ, van Montfrans JM, Hoogstraat $M$, et al. Target next-generation sequencing: a novel diagnostic tool for primary immunodeficiencies. J Allergy Clin Immunol 2014; 133:529-534.

30. Stoddard JL, Niemela JE, Fleisher TA, Rosenzweig SD. Target NGS: a costeffective approach to molecular diagnosis of PIDs. Front Immunol 2014; 5:531.

31. Saudi Mendeliome Group. Comprehensive gene panels provide advantages over clinical exome sequencing for Mendelian diseases. Genome Biol 2015; $16: 134$.

32. Majewski J, Schwartzentruber J, Lalonde E, et al. What can exome sequencing do for you? J Med Genet 2011; 48:580-589.

33. Boisson $B$, Wang YD, Bosompem A, et al. A recurrent dominant negative E47 mutation causes agammaglobulinemia and BCR(-) B cells. J Clin Invest 2013; 123:4781-4785.

34. Itan $Y$, Zhang SY, Vogt G, et al. The human gene connectome as a map of short cuts for morbid allele discovery. Proc Natl Acad Sci U S A 2013; 110:5558-5563.

35. Itan $Y$, Mazel M, Mazel B, et al. HGCS: an online tool for prioritizing diseasecausing gene variants by biological distance. BMC Genomics 2014; 15:256.

36. Itan $Y$, Shang $L$, Boisson $B$, et al. The human gene damage index as a genelevel approach to prioritizing exome variants. Proc Natl Acad Sci U S A 2015; 112:13615-13620.

37. Petrovski S, Wang $\mathrm{Q}$, Heinzen EL, et al. Genic intolerance to functional variation and the interpretation of personal genomes. PLoS Genet 2013; 9:e1003709.

38. Van Montfrans JM, Hartman EA, Braun KP, et al. Phenotypic variability in patients with ADA2 deficiency due to identical homozygous R169Q mutations. Rheumatology (Oxford) 2016; 55:902-910.

39. Chung LK, Park YH, Zheng $Y$, et al. The yersinia virulence factor YopM hijacks host kinases to inhibit type III effector-triggered activation of the pyrin inflammasome. Cell Host Microbe 2016; 20:296-306.

40. Sauna ZE, Kimchi-Sarfaty C. Understanding the contribution of synonymous mutations to human disease. Nat Rev Genet 2011; 12:683-691.
41. Davydov EV, Goode DL, Sirota $M$, et al. Identifying a high fraction of the human genome to be under selective constraint using GERP++. PLoS Comput Biol 2010; 6:e1001025.

42. Kumar P, Henikoff S, Ng PC. Predicting the effects of coding non-synonymous variants on protein function using the SIFT algorithm. Nat Protoc 2009; 4:1073-1081.

43. Adzhubei IA, Schmidt S, Peshkin L, et al. A method and server for predicting damaging missense mutations. Nat Methods 2010; 7:248-249.

44. Itan $Y$, Shang $L$, Boisson $B$, et al. The mutation significance cutoff: gene-level

- thresholds for variant predictions. Nat Methods 2016; 13:109-110.

The article describes the use of MSC to add gene specificity to a variant prediction tool, especially the CADD score.

45. Merico D, Roifman M, Braunschweig $U$, et al. Compound heterozygous mutations in the noncoding RNU4ATAC cause Roifman Syndrome by disrupting minor intron splicing. Nat Commun 2015; 6:8718.

46. Starokadomskyy P, Gemelli T, Rios JJ, et al. DNA polymerase- $\alpha$ regulates the activation of type I interferons through cytosolic RNA:DNA synthesis. Nat Immunol 2016; 17:495-504.

47. Redon $\mathrm{R}$, Ishikawa $\mathrm{S}$, Fitch $\mathrm{KR}$, et al. Global variation in copy number in the human genome. Nature 2006; 444:444-454.

48. Alkan C, Kidd JM, Marques-Bonet $\mathrm{T}$, et al. Personalized copy number and segmental duplication maps using next-generation sequencing. Nat Genet 2009; 41:1061-1067.

49. Freeman JL, Perry GH, Feuk L, et al. Copy number variation: new insights in genome diversity. Genome Res 2006; 16:949-961.

50. Sharp AJ, Locke DP, McGrath SD, et al. Segmental duplications and copynumber variation in the human genome. Am J Hum Genet 2005; 77:78-88.

51. Lupski JR. Genomic disorders: structural features of the genome can lead to DNA rearrangements and human disease traits. Trends Genet 1998; 14:417-422.

52. Itan $Y$, Bryson $K$, Thomas MG. Detecting gene duplications in the human lineage. Ann Hum Genet 2010; 74:555-565.

53. Dennis MY, Harshman L, Nelson BJ, et al. The evolution and population diversity of human-specific segmental duplications. Nat Ecol Evol 2017; 1:; pii: 0069.

54. Emanuel BS, Shaikh TH. Segmental duplications: an 'expanding' role in genomic instability and disease. Nat Rev Genet 2001; 2:791-800.

55. de Ravel TJ, Devriendt K, Fryns JP, Vermeesch JR. What's new in karyotyping? The move towards array comparative genomic hybridisation (CGH). Eur J Pediatr 2007; 166:637-643.

56. Shinawi M, Cheung SW. The array $\mathrm{CGH}$ and its clinical applications. Drug Discov Today 2008; 13:760-770.

57. Kazenwadel J, Secker GA, Liu YJ, et al. Loss-of-function germline GATA2 mutations in patients with MDS/AML or MonoMAC syndrome and primary lymphedema reveal a key role for GATA2 in the lymphatic vasculature. Blood 2012; 119:1283-1291.

58. Kadalayil L, Rafiq S, Rose-Zerilli MJ, et al. Exome sequence read depth methods for identifying copy number changes. Brief Bioinform 2015; $16: 380-392$.

The article provides assessment and comparison of the available read depth methods to identify CNVs from exome sequence data.

59. Tattini $L$, D'Aurizio $R$, Magi $A$. Detection of genomic structural variants from next-generation sequencing data. Front Bioeng Biotechnol 2015; 3:92.

60. Plagnol V, Curtis J, Epstein $M$, et al. A robust model for read count data in exome sequencing experiments and implications for copy number variant calling. Bioinformatics 2012; 28:2747-2754.

61. Sandilands A, Terron-Kwiatkowski A, Hull PR, et al. Comprehensive analysis of the gene encoding filaggrin uncovers prevalent and rare mutations in ichthyosis vulgaris and atopic eczema. Nat Genet 2007; 39:650-654.

62. D'Errico I, Gadaleta G, Saccone C. Pseudogenes in metazoa: origin and features. Brief Funct Genomic Proteomic 2004; 3:157-167.

63. Aradhya $S$, Bardaro $T$, Galgóczy $P$, et al. Multiple pathogenic and benign genomic rearrangements occur at a $35 \mathrm{~kb}$ duplication involving the NEMO and LAGE2 genes. Hum Mol Genet 2001; 10:2557-2567.

64. Fusco F, Paciolla M, Napolitano F, et al. Genomic architecture at the Incontinentia Pigmenti locus favours de novo pathological alleles through different mechanisms. Hum Mol Genet 2012; 21:1260-1271.

65. Bardaro T, Falco G, Sparago A, et al. Two cases of misinterpretation of molecular results in incontinentia pigmenti, and a PCR-based method to discriminate NEMO/IKKgamma dene deletion. Hum Mutat 2003; 21:8-11.

66. Notarangelo LD, Fleisher TA. Targeted strategies directed at the molecular

- defect: toward precision medicine for select primary immunodeficiency disorders. J Allergy Clin Immunol 2017; 139:715-723.

The article reviews all the known therapeutic strategies directly targeted at the molecular defects in the field of inborn errors of immunity. 